\title{
Clinical Efficacy of Tiletamine-Zolazepam and Ketamine-Diazepam Combination on Quality of Anesthesia for Ovariohysterectomy in Dog
}

\section{Prashant Dilip Salve ${ }^{1 *}$, Milind Gonduji Thorat ${ }^{2}$, Ratnakar Vasantrao Raulkar ${ }^{3}$, Ravindrakumar Imdeo Hatzade ${ }^{4}$, Mahesh V Ingawale ${ }^{5}$, Mahesh Babasaheb Pawar ${ }^{1}$ and Avinash Ganpat Jadhav ${ }^{1}$}

${ }^{1}$ M V Sc Student, Department of Veterinary Surgery and Radiology, Maharashtra, India

${ }^{2}$ Professor and Head, Department of Veterinary Surgery and Radiology,

Maharashtra, India

${ }^{3}$ Assistant Professor, Department of Veterinary Surgery and Radiology,

Maharashtra, India

${ }^{4}$ Department of Veterinary Medicine, Maharashtra, India

${ }^{5}$ Assistant Professor, Department of Veterinary Gynaecology and Obstetrics,

Maharashtra, India

*Corresponding Author: Prashant Dilip Salve, M V Sc Student, Department of

Veterinary Surgery and Radiology, Maharashtra, India.
Received: January 03, 2022

Published: January 31, 2022

(C) All rights are reserved by Prashant Dilip

Salve., et al.

\begin{abstract}
The present study was undertaken to compare the anesthetic efficacy of tiletamine-zolazepam and ketamine-diazepam combination in 12 bitches presented to the TVCC, PGIVAS, Akola for ovariohysterectomies. These 12 dogs were randomly divided into two equal groups. Group I dogs were anaesthetized with tiletamine-zolazepam and Group II dogs were anaesthetized with ketaminediazepam combination. In both the groups xylazine was used as a pre-anaesthetic. Quality of anaesthesia, assessment of anaesthesia and clinico-physiological parameters were recorded at different time intervals during the study period. The quality of anaesthesia was found to be superior in group I as compared to group II. Induction of anaesthesia was rapid and smooth in both the groups. However, duration of anaesthesia and recovery time was longer in group I as compared to group II. Smooth recovery was observed in both the groups. Rectal temperature and respiration rate was showing decreasing trend in both the groups however, these values were within normal physiological range. Heart rate in both the group was showing decreasing trend with the appearance of bradycardia during recovery in both the groups. $\mathrm{SpO}_{2}$ level was fluctuating within normal physiological limit in both the groups. Quality of muscle relaxation of tiletamine was better than ketamine and overall quality of anaesthesia of tiletamine-zolazepam was found to be superior as compare to ketamine-diazepam combination.
\end{abstract}

Keywords: Dog; Ovariohysterectomy; Tiletamine-Zolazepam; Ketamine-Diazepam; Xylazine

\section{Introduction}

Ovariohysterectomy of bitches is routinely performed as an animal birth control programme in most of the cities which needs to be undertaken under general anaesthesia. The surgical proce- dures are painful and therefore require an ideal anaesthetic protocol which produce sleep, amnesia, analgesia and muscle relaxation. General anaesthesia is defined as optimum induced drug intoxication of CNS which produces a reversible state of unconsciousness that provides immobilization, loss of sensation, analgesia and

Citation: Prashant Dilip Salve., et al. "Clinical Efficacy of Tiletamine-Zolazepam and Ketamine-Diazepam Combination on Quality of Anesthesia for Ovariohysterectomy in Dog". Acta Scientific Veterinary Sciences 4.2 (2022): 51-56. 
muscle relaxation [1]. Two critical stages of general anaesthesia are induction and recovery, therefore most of the life-threatening hazards can occur during these two stages. Hence, anaesthetic agent must possess a quality of short onset and smooth recovery from anaesthesia. An ideal anaesthetic drug should produce sleep, amnesia, analgesia and muscle relaxation [2]. However, all these characteristics cannot be provided by a single agent and therefore a combination of drugs is used. Every drug has some unfavourable effects and therefore the combination of various drugs is employed to reduce the side effects of a single drug and thus the anaesthesia is produced with more satisfactory outcomes [3]. Premedication should always include an analgesic drug to control the peri and post-operative pain associated with surgical procedures [4]. Xylazine and ketamine are the main anaesthetic agents that has been used in veterinary surgery. Xylazine is a $\alpha_{2}$-adrenoceptor agonist which has sedative and analgesic effect while the ketamine is a dissociative anesthetic agent. Diazepam belongs to the benzodiazepine group, acts as central muscle relaxant and it reduces the muscle tone associated with the ketamine [5]. The combination of ketamine-diazepam has been used in veterinary surgery as a dissociative anaesthetic agent. Ketamine possesses poor visceral analgesic property. However, it can be used in combination with xylazine and/or diazepam to provide good visceral analgesia in case of abdominal surgery including ovariohysterectomy [6]. Zoletil is a combination of tiletamine hydrochloride which belongs to the class dissociative anesthetics and zolazepam hydrochloride which belongs to the benzodiazepine group. The basic descriptive pharmacology of tiletamine (2-(ethylamino)-2-(2-thienyl) cyclohexanone hydrochloride) was first reported in 1969 [7]. Tiletamine when used alone, produces cataleptic dissociative state which is associated with muscle spasm and convulsive seizure. To avoid these side effects, it is often combined with zolazepam which is a tranquilizer in action. Basic pharmacology of zolazepam $\mathrm{HCl}$ is $4(-0$-fluorophenyl) 6,8-dihydro-1,3,8, trimethyl pyrazolo-3, $3 \mathrm{e}_{1}, 4$ diazepam-7(14)-one $\mathrm{HCl}$. The combination was found to be useful for the induction of anesthesia as well as a sole anesthetic agent for a wide variety of diagnostic and surgical procedures. In canids zolazepam metabolizes rapidly than the tiletamine and hence there can be transient tachycardia, athetoid movements and rough recovery $[8,9]$. Combination of Tiletamine-Zolazepam-Ketamine-Xylazine (TKX) has been used for the sterilization of street dogs in field clinics [10] and to compare TKX with tiletamine-zolazepam [11].

\section{Materials and Methods}

All the dogs selected for study were kept on fasting for 12 hours and water was with-held for 4 hours prior to surgery. The surgical site was prepared aseptically by clipping, shaving and scrubbing with antiseptic solution.

The dogs were randomly divided into two equal groups irrespective of age, breed and body weight. In group I induction was achieved using Tiletamine-Zolazepam combination @ $3 \mathrm{mg} / \mathrm{Kg}$ BW I/V and anaesthesia was maintained by intravenous administration of half of the induction dose of anaesthetic agent, whereas in group II induction was achieved using Ketamine @ $3 \mathrm{mg} / \mathrm{Kg} \mathrm{BW}$, I/V preceded by administration of Diazepam @ $0.25 \mathrm{mg} / \mathrm{kg} \mathrm{BW} \mathrm{I/V}$ and anaesthesia was maintained by administrating the same dose as that of induction dose of anaesthetic agent.

Quality of anaesthesia includes palpebral reflex, pedal pinch reflex, position of the eyeball and mandibular muscle tone was assessed and recorded during anaesthetic period. Assessment of anaesthesia includes induction time (seconds), duration of anaesthesia (minutes) and recovery time (minutes) was recorded. Induction time was recorded from the administration of induction dose of anaesthetic agent to the disappearance of body reflexes. Duration of anaesthesia was recorded from the disappearance of body reflexes to the return of first righting reflex and recovery time was recorded from the administration of last bolus of anaesthetic agent during the surgery to the return of first righting reflex. Clinico-physiological parameters includes rectal temperature, respiration rate, heart rate and $\mathrm{SpO}_{2}$ were recorded before sedation ( $0 \mathrm{~min}$ ), at 15, 30, 45 min and at the time of recovery from anaesthesia. Rectal temperature was recorded with the help of digital thermometer and was measured in degree Fahrenheit. Respiratory rate was recorded in breaths/minutes by observing the insufflation and deflation of thorax and abdominal wall. Heart rate was calculated from the lead II of ECG paper. The ECG was recorded at speed $50 \mathrm{~mm} / \mathrm{sec}$ and amplitude at $10 \mathrm{mV}$. Peripheral blood oxygen saturation level $\left(\mathrm{SpO}_{2}\right)$ was recorded with the help of NEW GEN MEDICAL SYSTEMS Pulse Oximeter by applying the probe to the upper lip of the dog.

The data was statistically analysed using the ICAR WASP 2.0 software's Two Way Factorial Experimental Design.

The Analysis of Variance (ANOVA) was used to compare the means of both the groups and different intervals.

Citation: Prashant Dilip Salve., et al. "Clinical Efficacy of Tiletamine-Zolazepam and Ketamine-Diazepam Combination on Quality of Anesthesia for Ovariohysterectomy in Dog". Acta Scientific Veterinary Sciences 4.2 (2022): 51-56. 


\section{Results and Discussion}

\section{Quality of anaesthesia}

Quality of anaesthesia was assessed by monitoring palpebral and pedal pinch reflex, position of eyeball and mandibular muscle tone and has been shown in table 1 .

\begin{tabular}{|l|c|c|}
\hline & Group I & Group II \\
\hline \multirow{2}{*}{ Palpebral reflex } & Weak (3) & Weak (3) \\
\cline { 2 - 3 } & Absent (3) & Absent (3) \\
\hline Pedal pinch reflex & Absent (6) & Absent (6) \\
\hline \multirow{2}{*}{ Eyeball position } & Central (5) & Central (6) \\
\cline { 2 - 3 } & Ventromedial (1) & \\
\hline Mandibular muscle tone & Excellent (6) & Excellent (3) \\
\hline & Moderate (0) & Moderate (3) \\
\hline
\end{tabular}

Table 1. Evaluation of body reflexes to assess quality of anaesthesia.

The number of animals with the various reflexes present or absent is indicated in parentheses.

In both the groups, palpebral reflex was weak in three dogs, whereas it was absent in three dogs. Presence of palpebral reflex in both the groups might be due to the characteristic of dissociative anaesthetic agent [12], whereas absence of it indicates that dogs were in a medium or deep plane of anaesthesia [12]. Pedal pinch reflex was absent in all dogs of both the groups. Absence of pedal pinch reflex indicates that quality of analgesia was excellent in both the groups. This might be due to visceral analgesia produced by the $\alpha-2$ agonist as the dissociative anaesthetic agents are poor analgesic. Xylazine acts on the CNS and inhibits interneural transmission which results into depression of CNS, muscle relaxation and visceral analgesia [13]. Eyeball was positioned centrally in all the dogs of both the groups, except in one dog from group I where it was positioned ventromedially at the time of recovery from anaesthesia. The eyeball does not rotate in the animals anaesthetized with dissociative anaesthetic agents. When the eyeball is located at centre, it indicates that the level of anaesthesia is deep, whereas when it is located ventromedially, it indicates that the level of anaesthesia is medium [12]. In all the dogs of group I the mandibular muscle tone was excellent and it was moderate in three dogs and excellent in three dogs of group II. Muscle hypertonus can be a feature of dis- sociative anaesthetic agents [12] and therefore benzodiazepines should be added to dissociative anaesthetic protocols. The quality of muscle relaxation was excellent in group I compared to group II. This might be due to the tiletamine having more muscle relaxation quality than ketamine [14]. The muscle relaxation was due to diazepam and zolazepam induced inhibition of internuncial neurons at the spinal level [15]. Similar observation regarding quality of anaesthesia has been reported by $[6,14,16-19]$.

\section{Assessment of anaesthesia}

Assessment of anaesthesia was evaluated on the basis of induction time, duration of anaesthesia and recovery time and has been shown in table 2.

\begin{tabular}{|l|c|c|c|}
\hline Group & $\begin{array}{c}\text { Induction Time } \\
\text { (seconds) }^{\text {NS }}\end{array}$ & $\begin{array}{c}\text { Duration of } \\
\text { Anesthesia } \\
\text { (Minutes) }^{*}\end{array}$ & $\begin{array}{c}\text { Recovery Time } \\
\text { (seconds)* }^{*}\end{array}$ \\
\hline Group I & $48.16 \pm 4.90$ & $125.33 \pm 12.52$ & $78.83 \pm 10.08$ \\
\hline Group II & $36.55 \pm 7.03$ & $88.00 \pm 9.35$ & $36.83 \pm 9.67$ \\
\hline
\end{tabular}

Table 2: Mean \pm SE of Induction Time (seconds), Duration of Anaesthesia (Minutes) and Recovery Time (Minutes).

* Significant at 5\%, NS - Non-Significant.

In group I, mean induction time was $48.16 \pm 4.90$ whereas it was $36.55 \pm 7.03$ in group II. Mean duration of anaesthesia and recovery time in group I was $125.33 \pm 12.52$ and $78.83 \pm 10.08$ respectively whereas in group II mean duration of anaesthesia and recovery time was $88.00 \pm 9.35$ and $36.83 \pm 9.67$ respectively. There was significant $(\mathrm{p}<0.05$ ) difference between the groups in duration of anaesthesia and recovery time. Longer period of duration of anaesthesia in group I could be due to the feature of tiletamine being more potent than ketamine and its duration of action is longer than ketamine $[5,14]$. Similar observations were recorded by $[6,14,20-22]$. Longer period of recovery time in group I as compared to group II might be due to the high potency of tiletamine compared with ketamine [5]. In dissociative anaesthetic recovery time is dose dependent. Higher dose of tiletamine-zolazepam was used by [23] and reported longer period of recovery time whereas lower dose of tiletamine-zolazepam was used by [18] and reported shorter period of recovery time. Ketamine was used at high dose by [6] and reported longer period of recovery time. 
Clinico-physiological parameters

Clinico-physiological parameters includes rectal temperature, respiration rate, heart rate and $\mathrm{SpO}_{2}$ were recorded at $15,30,45$ min and during recovery from anaesthesia and has been shown in table 3.

\begin{tabular}{|l|c|c|c|c|c|c|}
\hline & Group & \multicolumn{4}{|c|}{ Time (min) } \\
\hline & & $\mathbf{0}$ & $\mathbf{1 5}$ & $\mathbf{3 0}$ & $\mathbf{4 5}$ & At Recovery \\
\hline \multirow{2}{*}{ Rectal Temperature } & Group I & $101.8 \pm 0.29$ & $100.7 \pm 0.36^{\mathrm{NS}}$ & $99.65 \pm 0.46^{* *}$ & $99.07 \pm 0.51^{* *}$ & $99.43 \pm 0.39^{* *}$ \\
& Group II & $101.81 \pm 0.27$ & $100.9 \pm 1.03^{\mathrm{NS}}$ & $100.3 \pm 0.95^{* *}$ & $99.33 \pm 0.93^{* *}$ & $98.05 \pm 0.78^{* *}$ \\
\hline \multirow{2}{*}{ Respiration } & Group I & $25.50 \pm 3.27$ & $16.00 \pm 4.76^{* *}$ & $18.00 \pm 2.09^{* *}$ & $17.16 \pm 2.42^{* *}$ & $17.00 \pm 1.86^{* *}$ \\
Rate & Group II & $26.16 \pm 2.46$ & $14.16 \pm 0.74^{* *}$ & $15.00 \pm 1.09^{* *}$ & $16.83 \pm 1.62^{* *}$ & $20.161 .47^{* *}$ \\
\hline \multirow{2}{*}{ Heart Rate } & Group I & $124.50 \pm 9.34$ & $80.00 \pm 9.30^{* *}$ & $73.16 \pm 7.93^{* *}$ & $69.83 \pm 9.13^{* *}$ & $64.16 \pm 4.16^{* *}$ \\
& Group II & $107.00 \pm 8.79$ & $62.16 \pm 6.92^{* *}$ & $79.83 \pm 7.37^{* *}$ & $76.33 \pm 4.52^{* *}$ & $63.00 \pm 4.03^{* *}$ \\
\hline \multirow{2}{*}{$\mathrm{SpO}_{2}$} & Group I & $98.50 \pm 0.76$ & $94.66 \pm 0.71^{* *}$ & $95.16 \pm 1.13^{* *}$ & $95.50 \pm 0.84^{* *}$ & $98.33 \pm 0.71^{\text {NS }}$ \\
& Group II & $98.66 \pm 0.33$ & $95.16 \pm 0.70^{* *}$ & $95.50 \pm 0.50^{* *}$ & $95.66 \pm 0.95^{* *}$ & $97.00 \pm 1.48^{\text {NS }}$ \\
\hline
\end{tabular}

Table 3: Mean $\pm \mathrm{SE}$ of Rectal Temperature $\left({ }^{\circ} \mathrm{F}\right)$, Respiration Rate (breaths $\left./ \mathrm{min}\right)$, Heart Rate (beats $\left./ \mathrm{min}\right)$ and $\mathrm{SpO}{ }_{2}(\%)$.

$$
\text { ** Significant at } 1 \% \text { level, NS-Non significance. }
$$

Rectal Temperature was decreased significantly $(\mathrm{p}<0.01)$ at $30 \mathrm{~min}, 45 \mathrm{~min}$ and during recovery from anaesthesia in both the groups when compared to base value $(0 \mathrm{~min})$. The decreased in rectal temperature might be attributed to depression of thermoregulatory centre in the hypothalamus $[6,24,25]$ or due to muscle relaxation, decreased in metabolic rate and thermoregulatory centre depression as a sequel of anaesthesia [26,27]. The observations regarding rectal temperature are comparable with the observations reported by [6,24-28].

Respiration rate was decreased significantly $(\mathrm{p}<0.01)$ at 15 , $30,45 \mathrm{~min}$ and during recovery from anaesthesia in both the groups when compared to base value ( $0 \mathrm{~min})$. The respiration rate in both the groups was depressed could be due to depression of respiratory centre located in medulla oblongata by anaesthetic agents $[6,16,27,29]$. Similar observation has also been reported by $[24,27,29,30]$ where they found significant declined in respiration rate. However, [6] in their study reported that respiration rate decreased non-significantly. Non-significant difference in the respiratory rate between the two groups was recorded by [16].

In both the groups heart rate was declined significantly $(\mathrm{p}<$ 0.01 ) at 15,30 and $45 \mathrm{~min}$ when compared with base value ( $0 \mathrm{~min}$ ). Bradycardia was observed in both the groups during recovery from anaesthesia. The heart rate might be decreased due to depression of sympathetic activity and increase in parasympathetic effect on virtue of $\alpha 2$ agonist's property [31]. This ubiquitous significantly reduced heart rate could be due to the anaesthetic effect which inhibits the CNS and excites the vagus nerve [32] and eventually causes the drop-in heart rate. Furthermore, the anaesthetic agent and sedative can simultaneously suppress the cardiac electrical stimulation [22]. These findings related to heart rate are corroborated with the findings reported by [16,22,28,31-33]. However, [6] in their study observed that decreased in heart rate was nonsignificant.

$\mathrm{SpO}_{2}$ level was decreased significantly $(\mathrm{p}<0.01)$ in both the groups at 15, 30 and 45 min when compared with its base value $(0$ min) but during recovery from anaesthesia it was elevated in both the groups. However, $\mathrm{SpO}_{2}$ level was found to be fluctuating within normal physiological range throughout anaesthetic period in both the groups. The marginal decreased in the value of $\mathrm{SpO}_{2}$ during anaesthesia might be attributed to the effect of xylazine which reduced the depth of respiration, but not the rate, leading to lowered oxygen saturation values [34] or it might be due to the anaesthetic agent's effects on the ventilator function of the lungs. Low $\mathrm{SpO}_{2}$ indicates a decreased in peripheral oxygenation and tissue perfusion [25]. The observations related to the $\mathrm{SpO}_{2}$ in this study are in accordance with $[18,24,27]$. 


\section{Conclusion}

Duration of anaesthesia and recovery time is longer in tiletamine-zolazepam combination compared with ketamine-diazepam combination. However, smooth recovery was observed without crying in both the anaesthetic protocols. Muscle relaxation was excellent in group I whereas it was moderate in group II. Therefore, it can be concluded that muscle relaxation quality of tiletaminezolazepam combination is superior than ketamine-diazepam combination.

\section{Bibliography}

1. Ilievska K., et al. "Comparison of the Anesthetic effects of Xylazine/Ketamine, Propofol and Zoletil in Dogs". Days of Veterinary Medicine 2012, $3^{\text {rd }}$ International Scientific Meeting (2012): 40-43.

2. Jena B., et al. "Clinical evaluation of total intravenous anesthesia using xylazine or dexmedetomidine with propofol in surgical management of canine patients". Veterinary World 7.9 (2014): 671-680.

3. Martinez-Taboada F and EA Leece. "Comparison of propofol with ketofol, a propofol - ketamine admixture, for induction of anesthesia in healthy dogs". Veterinary Anesthesia and Analgesia 41.6 (2014): 575-582.

4. Bednarski RM. "Anesthesia, Analgesia and Immobilization of Dogs and Cats". In: Tranquilli W.J., Thurmon JC and Grimm KA. Veterinary Anaesthesia and Analgesia. $4^{\text {th }}$ Edition. Iowa USA: Blackwell Publishing Ltd. (2007): 705-715.

5. Lin HC. "Dissociative Anaesthetics". In: Tranquilli W.J., Thurmon J.C. and Grimm K.A. (edited). Lumb and Jones. Veterinary Anesthesia and Analgesia. $4^{\text {th }}$ ed. Iowa USA: Blackwell Publishing Ltd. (2007): 301-304.

6. Yohannes G., et al. "Clinical Evaluation of Anesthetic Combinations of Xylazine-Ketamine, Diazepam-Ketamine and Acepromazine-Ketamine in Dogs of Local Breed in Mekelle, Ethiopia". SOJ Dairy and Veterinary Science 4.2 (2018): 1-9.

7. Chen G., et al. "The pharmacology of 2-(Ethylamino)-2-(2Thienyl)- Cyclohexamine-HCI (CI-634)". Journal of Pharmacology and Experimental Therapeutics 168.1 (1969): 171-179.

8. Dugassa J and A Fromsa. "Review on Dissociative Anaesthetics and Compatible Drug Combinations in Veterinary Clinical Practice". Veterinary Medicine Open Journal 3.1 (2018): 21-30.
9. Kastner SBR. "Intravenous anaesthetics". In: C. Seymour and T. Duke-Novakovski (edited). BSAVA Manual of Canine and Feline Anesthesia and Analgesia. $2^{\text {nd }}$ ed. Gloucester: British Small Animal Veterinary Association Publication (2007): 142-143.

10. Landry J and P Maza. "Effectiveness of the anesthetic combination of tiletamine, zolazepam, ketamine and xylazine for the sterilization of street dogs in field clinics". Veterinary Record Case Reports 8.2 (2020): e000953.

11. Chen HC., et al. "Comparison of Tiletamine-Zolazepam and Tiletamine-Zolazepam-Ketamine-Xylazine Administered Intravenously in Dogs". Malaysian Veterinary Journal 17.1 (2005): $1-6$.

12. Haskins SC. "Monitoring Anesthetized Patients". In: Tranquilli W.J., Thurmon J.C. and Grimm K.A. (editors). Lumb \& Jones' Veterinary Anaesthesia and Analgesia. 4th ed. Iowa USA: Blackwell Publishing Ltd. (2007): 533-558.

13. Williams LS., et al. "Use of the anesthetic combination of tiletamine, zolazepam, ketamine, and xylazine for neutering feral cats". Journal of the American Veterinary Medical Association 220.10 (2002): 1491-1495.

14. Phutthachalee S., et al. "Comparison of the Anesthetic Effects of Tiletamine HCl-Zolazepam-Xylazine and Ketamine-Diazepam-Xylazine in Older Foals under Field Conditions". Kasetsart Journal - Natural Science 46.2 (2012): 190-199.

15. Hall LW., et al. "Principle of Sedation, Analgesia and Premedication”. In: Veterinary Anaesthesia. 10 ${ }^{\text {th }}$ ed. London: Harcourt Publishers Ltd. (2001): 82-83.

16. Samsuddi S. "Comparison of Xylazine and Dexmedetomidine as Pre-Anaesthetic for Ketamine Anaesthesia for Various Surgeries in Dogs". M. V. Sc thesis submitted to Karnataka Veterinary, Animal and Fisheries Sciences University, Bidar. The Indian journal of animal sciences 84.1 (2014): 8-12.

17. Shandilya M. "Studies on Clinical Efficacy of Midazolam-Ketamine and Xylazine-Ketamine Anaesthesia in Canines". M. V. Sc thesis submitted to Rajasthan University of Veterinary and Animal Sciences, Bikaner (2016).

18. Romagnoli N., et al. "Short-term general anaesthesia with tiletamine/zolazepam in horses sedated with medetomidine for castration under field conditions". Journal of Equine Veterinary Science 67 (2018): 50-54. 
19. Rydén A., et al. "Physiological and Clinical Responses in Pigs in Relation to Plasma Concentrations during Anesthesia with Dexmedetomidine, Tiletamine, Zolazepam, and Butorphanol". Animals 11.6 (2021): 1482.

20. Haigh JC., et al. "Immobilization of Polar Bears (Ursus Maritimus Phipps) with a Mixture of Tiletamine Hydrochloride and Zolazepam Hydrochloride". Journal of Wildlife Diseases 21.1 (1985): 43-47.

21. Nam SW., et al. "Anesthetic and Cardiopulmonary Effects of Butorphanol-Tiletamine-Zolazepam-Medetomidine and Tramadol-Tiletamine-Zolazepam-Medetomidine in Dogs". Journal of Veterinary Clinics 30.6 (2013): 421-427.

22. Liang Y., et al. "Comparison of the anesthesia effects of ketamine, dexmedetomidine and tiletamine-zolazepam with or without tramadol in cats". Medycyna Weterynaryjna77.8 (2021): 413-418.

23. Rajankutty K. "General Anaesthesia in Dogs with TiletamineZolazepam". Ph. D. thesis submitted to College of Veterinary and Animal Sciences, Mannuthy, Thrissur (1995).

24. Pereira SARS., et al. "Anesthetic quality and cardiovascular and respiratory effects of continuous intravenous infusion of tiletamine-zolazepam in bitches". Pesquisa Veterinária Brasileira 39.3 (2019): 214-220.

25. Munif MR., et al. "Pulse oximetry and clinical changes during electrosurgery in dogs anaesthetized with Xylazine-Thiopentone and Xylazine-Ketamine combinations". Research in Agriculture Livestock and Fisheries 7.1 (2020): 97-105.

26. Lu DZ., et al. "A comparison of anaesthetic and cardiorespiratory effects of tiletamine-zolazepam/xylazine and tiletaminezolazepam/xylazine/tramadol in dogs". Pakistan Veterinary Journal 34.1 (2014): 63-67.

27. Saikia A. "Romifidine and its Combination with Ketamine in Dog”. M. V. Sc thesis submitted to Assam Agricultural University, Khanapara, Guwahati (2016).

28. Lee JY., et al. "Antagonistic effect of flumazenil on tiletaminezolazepam-induced anaesthesia in Beagle dogs". Veterinarni Medicina 63.12 (2018): 555-560.

29. Ibrahim A. "Evaluation of Total Intravenous Anaesthesia by Ketamine-Xylazine Constant Rate Infusion in Dogs: A Novel Preliminary Dose Study". Veterinary Medicine Open Journal 2.2 (2017): 38-44.
30. Turunen H., et al. "Effects of Intramuscular Vatinoxan (MK467), Co- Administered with Medetomidine and Butorphanol, on Cardiopulmonary and Anaesthetic Effects of Intravenous Ketamine in Dogs". Veterinary Anaesthesia and Analgesia 47.5 (2020): 604-613.

31. Yaygingül R and A Belge. "The comparison of clinical and cardiopulmonary effects of xylazine, medetomidine and detomidine in dogs". Ankara Üniversitesi Veteriner Fakültesi Dergisi 65.3 (2018): 313-322.

32. Kwon YS., et al. "Comparison of Tiletamine/Zolazepam, Xylazine-Tiletamine/Zolazepam and Medetomidine-Tiletamine/ Zolazepam Anesthesia in Dogs". Journal of Veterinary Clinics 20.1 (2003): 33-41.

33. Lee JY., et al. "Comparison of anaesthetic and cardiorespiratory effects of xylazine or medetomidine in combination with tiletamine/zolazepam in pigs". Veterinary Record 167.7 (2010): 245-249.

34. Lewandowski AH., et al. "Tiletamine-Zolazepam, Ketamine, and Xylazine Anesthesia of Captive Cheetah (Acinonyx Jubatus)". Journal of Zoo and Wildlife Medicine 33.4 (2002): 332336.

\section{Assets from publication with us}

- Prompt Acknowledgement after receiving the article

- Thorough Double blinded peer review

- Rapid Publication

- Issue of Publication Certificate

- High visibility of your Published work

Website: www.actascientific.com/

Submit Article: www.actascientific.com/submission.php Email us: editor@actascientific.com

Contact us: +919182824667 